\title{
Intervenciones para mejorar el grado de cumplimiento de la higiene de manos en los servicios de urgencias
}

\author{
Interventions to improve hand hygiene compliance in \\ emergency departments
}

\author{
Pablo Chico Sánchez ${ }^{1}$ \\ ${ }^{1}$ Instituto de Investigación Sanitaria y Biomédica de Alicante ISABIAL. Alicante, España.
}

\section{Resumen}

Este trabajo es un comentario del artículo: Seo HJ, Sohng KY, Chang SO, Chaung SK, Wone JS, Choi MJ. Interventions to improve hand hygiene compliance in emergency departments: a systematic review. Journal of Hospital Infection. 2019;102(4):394-406. doi:10.1016/j. jhin.2019.03.013

\begin{abstract}
This text is a commentary on the article: Seo HJ, Sohng KY, Chang SO, Chaung SK, Wone JS, Choi MJ. Interventions to improve hand hygiene compliance in emergency departments: a systematic review. Journal of Hospital Infection. 2019;102(4):394-406. doi:10.1016/j. jhin.2019.03.013
\end{abstract}

Sección coordinada por

Consol Serra (consol.serra@upf.edu) I Ma del Mar Seguí (mm.segui@ua.es)

Fechas · Dates

Recibido: 2020.09.02 Aceptado: 2020.10.12 Publicado: 2020.11.04

\section{Correspondencia $\cdot$ Corresponding Author}

Pablo Chico Sánchez

Instituto de Investigación Sanitaria y Biomédica de Alicante ISABIAL. Unidad de Epidemiología. Servicio de Medicina Preventiva. Hospital General Universitario de Alicante.

chico_pab@gva.es 


\section{Resumen del artículo comentado}

La problemática sobre la higiene de manos en los servicios de urgencias es importante debido a que los procedimientos que en él se realizan son con frecuencia invasivos y de alto riesgo. Hasta la fecha, no ha habido revisiones exhaustivas acerca del grado de cumplimiento de la higiene de manos en dichos servicios. El objetivo de este estudio fue investigar el grado de cumplimiento de la higiene de manos (GCHM), los factores que influyen en su cumplimiento y las estrategias de intervención para su mejora en los servicios de urgencias. Para llevarlo a cabo, se utilizaron diversas bases de datos electrónicas para buscar artículos publicados desde 1948 hasta enero de 2018. Las bases de datos consultadas fueron ovidMEDLINE, ovidEMBASE, la Biblioteca Cochrane, CINAHL, Koreamed y Kmbas y se incluyeron todos los tipos de diseño de estudios. Dos revisores obtuvieron de forma independiente los datos y evaluaron los posibles sesgos utilizando para ello herramientas válidas y adecuadas. Se realizó una revisión narrativa incluyendo 24 estudios, de los cuales 12 fueron estudios transversales y 12 estudios de intervención. De los 12 estudios de intervención revisados, sólo el 33\% ( $n=4)$ tenía GCHM superiores al 50\%. Los factores asociados que influyeron en el GCHM fueron el tipo de profesional sanitario, indicación de la higiene de manos, grado de ocupación del servicio de urgencias, actitud positiva del personal sobre el cumplimiento de la higiene de manos, ubicación de los pacientes, existencia de observación sobre higiene de manos y turno de trabajo de los profesionales. Casi todos los estudios (83,3\%) aplicaron intervenciones multimodales o duales con el objetivo de mejorar el GCHM. Diversas estrategias, entre ellas la formación, la monitorización, el feedback, las campañas de concienciación y el establecimiento de recomendaciones, mejoraron de manera efectiva el GCHM. Los hallazgos de la revisión indican que hay margen de mejora en el GCHM en los servicios de urgencias. Se necesitan futuros estudios aleatorizados para determinar qué intervenciones son las más eficaces y a la vez sostenibles para mejorar el GCHM.

\section{Comentario}

La higiene de manos es la medida más importante para la prevención de las Infecciones Asociadas a los Cuidados de la Salud (IACS)(1-2). Dicha medida, es el pilar básico de lo que denominamos las Precauciones Estándar dentro de los Programas de Prevención y Control de Infecciones. El objetivo de estos programas es doble, tanto prevenir la aparición de infecciones en los pacientes como en los profesionales sanitarios. En la literatura encontramos multitud de estudios que avalan la importancia del adecuado cumplimiento de las recomendaciones sobre la higiene de manos por parte de los profesionales sanitarios, y la relación entre la mejora del grado de cumplimiento y la disminución de la incidencia de las IACS ${ }^{(3-6)}$.

Pese a todo este conocimiento, y con datos basados en la evidencia científica, el grado de cumplimiento de la higiene de manos sigue siendo muy variable. Los valores varían en función del área que estudiemos: urgencias, pediatría, unidades de críticos, etc. Los datos de los estudios revisados por los autores concuerdan 
con los que ha obtenido nuestro grupo de investigación, y son congruentes tanto en los factores asociados al grado de cumplimiento como en los resultados de su evaluación.

El patrón de oro para evaluar el grado de cumplimiento de la higiene de manos es la observación directa, siendo fundamental establecer un cronograma en los centros sanitarios que permita, mediante un sistema de vueltas, llegar a realizar la observación en todas las unidades del centro. Existe además gran variabilidad en los tiempos de observación, oscilando desde periodos que van de 20-30 min a periodos que se extienden hasta varias horas ${ }^{(9)}$. Las guías de la OMS recomiendan la observación durante periodos de 20 minutos (+10 minutos si fuese necesario $\left.{ }^{(1)}\right)$. Sin embargo, los períodos cortos de tiempo, pueden no reflejar el comportamiento del resto de la jornada de los profesionales sanitarios ${ }^{(10)}$, recomendándose por lo tanto un tiempo óptimo de observación de una hora en cada área o servicio evaluado(11).

Sin embargo, no sólo tenemos que quedarnos en la observación directa, sino que tenemos que ir un paso más allá. Aportar feedback y comunicar los resultados al personal sanitario, tanto a nivel individual como grupal, es fundamental para conseguir éxito en la mejora del grado de cumplimiento. Estudios como el de Arise et al.(12), concluyeron que, el uso de la observación directa y la aportación de feedback, son estrategias efectivas que consiguieron una mejora en el grado de cumplimiento de la higiene de manos. Dicha mejora, se mantuvo en el tiempo durante 5 años después de la finalización de dicho estudio.

Cabe destacar, que respecto a las oportunidades en las que se realiza la higiene de manos y considerando los 5 momentos de la OMS (1: antes de tocar al paciente, 2: antes de realizar una tarea limpia/aséptica, 3: después del riesgo de exposición a líquidos corporales, 4: después de tocar al paciente, 5: después del contacto con el entorno del paciente), existen diferencias significativas entre ellas. Hemos visto que el grado de cumplimiento es más alto en aquellas actividades que tienen lugar en los momentos "después" ( 3,4 y 5 de la OMS), mientras que es menor en los momentos "antes" ( 1 y 2 de la OMS) ${ }^{(8)}$. Esto pone de manifiesto la necesidad de un cambio de paradigma en los profesionales sanitarios que consideran la higiene de manos como una forma de protegerse después de entrar en contacto con el paciente. Hay que incidir en el papel que los sanitarios pueden ejercer como fuente de infección sobre los pacientes, quedando en sus manos evitar que lo sean.

En la revisión bibliográfica que nos ocupa, se destacan como factores asociados al cumplimiento, la categoría profesional de los sanitarios, el nivel de saturación del servicio de urgencias, la actitud positiva del personal sobre la higiene de manos, la ubicación de los pacientes, la existencia o no de observación directa, incluso el turno de trabajo de los profesionales. La mayoría de ellos no son modificables, máxime cuando en los servicios de urgencias, la afluencia de pacientes no es algo que pueda ser fácilmente controlable.

Por todo lo anterior, debemos de focalizar nuestros esfuerzos en aquellos factores que se consideren modificables, potenciando el conocimiento de los profesionales sobre cuándo y cómo realizar una adecuada higiene de manos, así como favo- 
recer su concienciación y motivación. Dichos aspectos son elementos claves para aumentar el GCHM. El estudio de Pittet et al. ${ }^{(13)}$, centrado en realizar intervenciones educativas y de sensibilización de los profesionales, mostró aumentos considerables y significativos en el grado de cumplimiento cuando se realizaban dichas intervenciones. En esa misma línea, otro factor asociado y que es claramente modificable, es la disponibilidad de botellas de solución alcohólica en formato bolsiIlo. Su presencia, ha mostrado un efecto favorecedor e independiente en relación con la realización de la higiene de manos. En un estudio de intervención en el que se proporcionó al personal evaluado solución alcohólica en formato bolsillo, se observó un efecto positivo, incrementando el GCHM hasta en un 20\%(14).

Las estrategias de Prevención y Control de Infecciones son elementos claves. Los programas que en ellas se desarrollan, han demostrado mediante una sólida evidencia científica, que son la piedra angular para hacer que el entorno sanitario sea un lugar seguro tanto para profesionales, como para pacientes. La higiene de manos es la estrategia fundamental, sin embargo, nuestros esfuerzos tienen que ir encaminados a evaluar, de manera constante en el tiempo, el grado de cumplimiento de higiene de manos por parte de los profesionales sanitarios en los 5 momentos en los que la OMS recomienda su realización. Teniendo en cuenta el papel que los profesionales sanitarios desempeñan como agentes de salud, tanto en el ámbito sanitario como en el de la comunidad, se hace necesario concienciarles de la importancia de una adecuada realización de higiene de manos ${ }^{(15)}$. Sólo de esa manera, se podrá garantizar un entorno sanitario seguro que consiga prevenir la aparición de las Infecciones Asociadas a los Cuidados de la Salud.

\section{Biliografía}

1. World Health Organization. WHO guideliness on hand hygiene in health care. 2009. Disponible en: https://apps.who.int/iris/bitstream/handle/10665/44102/9789241597906_eng.pdf?sequence=1.

2. Boyce J, Pittet D. Guideline for hand hygiene in health-care settings: Recommendations of the healthcare infection control practices advisory committee and the hicpac/shea/apic/idsa hand hygiene task force. Am J Infecti Control. 2002;30(8):S1-S46

3. Siegel JD, Rhinehart E, Jackson M, Chiarello L. 2007 Guideline for isolation precautions: preventing transmission of infectious agents in health care settings. Am J Infect Control. 2007;35(10):S65-S164

4. Chen YC, Sheng WH, Wang JT, Chang SC, Lin HC, Tien KL, et al. Effectiveness and limitations of hand hygiene promotion on decreasing healthcare-associated infections. PLoS One. 2011;6:e27163.

5. Akyol A, Ulusoy H, Ozen I. Handwashing: a simple, economical and effective method for preventing nosocomial infections in intensive care units. J Hosp Infect. 2006;62:395-405. 
6. Whitby $M$, Pessoa-Silva $C L$, McLaws $M L$, Allegranzi $B$, Sax H, Larson E, et al. Behavioural considerations for hand hygiene practices: the basic building blocks. J Hosp Infect. 2007;65:1-8.

7. Sánchez-Payá J, Galicia-García MD, Gracia-Rodríguez RM, García-González C, Fuster- Pérez M, López-Fresneda N, et al. Grado de cumplimiento y determinantes de las recomendaciones sobre la higiene de manos. Enferm Infecc Microbiol Clin. 2007;25:369-75

8. Gras-Valentí P, Mora-Muriel JG, Fuster-Pérez M, Benito Miralles CM, Vela-Morales MC, González-Hernández M, et al. Evolution and associated factors of hand hygiene compliance in a pediatric tertiary hospital. Am J Infect Control. 2020. DOI: 10.1016/j.ajic.2020.05.013

9. McAteer J, Stone S, Fuller C, Charlett A, Cookson B, Slade R, et al. Development of an observational measure of healthcare worker hand-hygiene behaviour: the handhygiene observation tool (HHOT). J Hosp Infect. 2008;68:222-9.

10. Stone S, Fuller C, Michie S, McAteer J, Charlett A. What Is the Optimal Period for Measuring Hand Hygiene Compliance: Are Longer Periods Better than 20-Minute Periods? Infection Control and Hospital Epidemiology. 2012;33(11):1174-6

11. Pittet D, Allegranzi B, Boyce J, World Health Organization World Alliance for Patient Safety First Global Patient Safety Challenge Core Group of Experts. The World Health Organization Guidelines on Hand Hygiene in Health Care and their consensus recommendations. Infect Control Hosp Epidemiol. 2009;30(7):611-22.

12. Arise K, Nishizaki S, Morita T, Yagi Y, Takeuchi S. Continued direct observation and feedback of hand hygiene adherence can result in long-term improvement. Am J Infect Control. 2016;44(11):e211-4.

13. Pittet D, Boyce J. Hand hygiene and patient care: pursuing the Semmelweis legacy. The Lancet Infectious Diseases. 2001;1:9-20

14. Nyamadzawo A, Nishio J, Okada S, Nyamakura R. Effect of using portable alcohol-based handrub on nurses' hand hygiene compliance and nasal carriage of staphylococcus aureus in a low-income health setting. Am J Infect Control. 2020;48(5):473-479.

15. Seo HJ, Sohng KY, Chang SO, Chaung SK, Wone JS, Choi MJ. Interventions to improve hand hygiene compliance in emergency departments: a systematic review. Journal of Hospital Infection. 2019;102(4):394-406. doi:10.1016/j.jhin.2019.03.013 